\title{
Los Procesos De Selección De Docentes Universitarios En La Universidad Contemporánea Y Su Relevancia Para Dar Respuesta A Las Misiones De Esta Institución
}

\author{
Diego Estevam Teleginski (Master) \\ Carina Rau (Master) \\ Décio Estevão do Nascimento (PhD) \\ Universidad Tecnológica Federal del Paraná, Curitiba, Brasil
}

Doi:10.19044/esj.2019.v15n4p400～URL:http://dx.doi.org/10.19044/esj.2019.v15n4p400

\section{Resumen}

La universidad contemporánea presenta una mayor interacción con la sociedad, en la atención de las demandas de públicos diferentes, lo que remite a un foco mayor en su tercera misión, o sea, el intercambio de conocimientos y tecnología. La universidad cuenta con la competencia de sus profesores para la enseñanza, investigación y vinculación universitaria y por eso resulta importante el proceso de selección de docentes que van a componer el cuadro de personal. El objetivo de este trabajo es demostrar lo relevante que es vinculación en los procesos de selección de docentes en dos universidades públicas brasileñas. Se asignaron expectativas en relación a la universidad contemporánea $\mathrm{y}$, a partir de eso, se identificaron criterios para relacionar lo que se espera de la tercera misión de la universidad y su proceso de selección del cuerpo docente. Con estos criterios se analizó la previsión de competencias en tercera misión en las convocatorias de docentes de las dos universidades seleccionadas. El método de análisis de contenido fue utilizado en la revisión bibliográfica y en el levantamiento de las categorías con las expectativas sobre la vinculación universitaria. Estas categorías sirvieron de base para el análisis de las convocatorias de docentes de las universidades. Los resultados apuntan que los procesos de incorporación de personal docente no exigen que el candidato tenga competencias en actividades de la tercera misión, sólo necesitando demostrar experiencia en la enseñanza y la investigación. Es éste el perfil de docente que estas instituciones seleccionan, incluso la vinculación siendo una de las tres misiones de la universidad. Seleccionado sin sesgo en la tercera misión el docente tiende a seguir una carrera apoyada solamente en la enseñanza y la investigación. 
Palabras clave: Universidad contemporánea. Vinculación Universitaria. Selección de docentes

\title{
The Processes of Selection of Professors in the Contemporary University and its Relevance to Respond to the Missions of this Institution
}

\author{
Diego Estevam Teleginski (Master) \\ Carina Rau (Master) \\ Décio Estevão do Nascimento (PhD)
}

Universidad Tecnológica Federal del Paraná, Curitiba, Brasil

\begin{abstract}
The contemporary university presents a greater interaction with society, in meeting the demands of different audiences, which refers to a greater focus in its third mission, that is, the exchange of knowledge and technology. The university has the competence of its professors for teaching, research and university linkage and that is why the process of selecting teachers who will compose the staff table is important. The objective of this paper is to demonstrate the relevance that is linked in the processes of teacher selection in two Brazilian public universities. Expectations were assigned in relation to the contemporary university and, from that, criteria were identified to relate what is expected of the third mission of the university and its process of selection of the faculty. With these criteria, the forecast of competencies in the third mission was analyzed in the calls for teachers from the two selected universities. The method of content analysis was used in the bibliographic review and in the lifting of the categories with the expectations regarding the university link. These categories served as the basis for the analysis of university calls for teachers. The results indicate that the processes of incorporation of teaching staff do not require the candidate to have competencies in activities of the third mission, only needing to demonstrate experience in teaching and research. This is the teacher profile that these institutions select, including the link being one of the three missions of the university. Selected without bias in the third mission the teacher tends to pursue a career supported only in teaching and research.
\end{abstract}


Keywords: Contemporary university, Third mission, Selection of teachers

\section{Introducción}

La universidad contemporánea incorporó como una de sus responsabilidades impactar en el desarrollo socioeconómico de la región por la relevancia de sus conocimientos e investigaciones (Etzkowitz, 1998, Etzkowitz y Leydesdorff, 2000, Rolfo y Finardi, 2014, Sam y Van der Sijde, 2014). Esta mayor interacción con el medio externo, generando contribuciones a la sociedad, compromiso con las cuestiones públicas, los servicios y las transferencias de tecnologías se define como tercera misión de la universidad (Gimenez, 2017, Sánchez-Barrioluengo, 2014, Vargiu, 2014).

Se vive una época de transición, que rompe con la producción segregada de conocimiento en la universidad y hace más fluida la relación entre enseñanza, investigación y extensión (Molas-Gallart, Salter, Patel, Scott y Duran, 2002). El énfasis está en la interacción de la universidad con la sociedad, atendiendo a las diversas demandas de públicos diferentes, al mismo tiempo que busca encajar la vinculación junto con las tradicionales enseñanza e investigación (D'este, Tang, Mahdi, Neely y SánchezBarrioluengo, 2013, Wang, Hu, Li y Pan, 2016).

Con estos roles y desafíos de la universidad actual, se entiende relevante discutir el desempeño de las universidades. Son los funcionarios que hacen la institución se desarrollar (Cascio, 2015, Pires y Rausch, 2016) y, por lo tanto, es importante seleccionar de manera adecuada los docentes e investigadores alineados con los intereses de la universidad (Cascio, 2015, Rodrigues y Veloso, 2013, Shipton, West, Dawson, Birdi y Patterson, 2006). El nivel de competencia del cuadro de docentes y su adherencia a las capacidades exigidas por la institución universitaria pueden impactar en el cumplimiento de los objetivos de ésta (Chiavenato, 2014, Gurgel, Pimenta, Santos y Medeiros Júnior, 2018), y por eso, la selección del personal de la universidad está entre las principales responsabilidades del sector de gestión de personas, pues impactan en el desempeño de la institución (Rana y Malik, 2017). El proceso de selección de personas para las organizaciones es muy importante, pues selecciona funcionarios capacitados para responder a las demandas de las instituciones (Gurgel et al., 2018, Rana y Malik, 2017), de acuerdo con sus planes futuros de crecimiento (Chiavenato, 2014).

Apoyándose en un análisis del contexto presentado, la pregunta que orienta este estudio es: ¿Cómo los procesos de selección de docentes evidencian la relevancia de las misiones de la universidad, de acuerdo con el papel de la universidad contemporánea?

El objetivo de este trabajo es demostrar lo relevante que es la tercera misión en los procesos de selección de docentes en dos universidades públicas brasileñas. En la delimitación de esta investigación se optó por 
seleccionar la Universidad Tecnológica Federal de Paraná (UTFPR), por ser la institución que los autores de la investigación están vinculados y por el interés en investigar cómo el proceso de selección de los docentes ocurre en la UTFPR. También fue seleccionada para el análisis la Universidad del estado de Campinas (Unicamp) por ser considerada la mejor universidad de América Latina, en 2017 y 2018, según el ranking de la Times Higher Education (THE) (https://www.timeshighereducation.com/, recuperado el 24, julio, 2018).

Se investigó la manera en que estas universidades contemplan la actuación en la extensión en el proceso de selección de personal docente. Las bases de informaciones, entendidas en su conjunto como instrumentos de gestión del cuerpo docente, son las convocatorias de los docentes.

\section{Los Desafíos de las Universidades Contemporáneas}

La universidad contemporánea tiene un papel complejo con una serie de expectativas que se le asignan: los estudiantes quieren una educación de calidad, los gobiernos anhelan un papel activo en el desarrollo regional y la economía, la sociedad espera la solución de muchos problemas para la universidad y las industrias buscan ideas e innovaciones para sus actividades de negocio (D'Este et al., 2013, Minola, Donina y Meoli, 2016). Varios cambios se produjeron en la política, la economía, el medio ambiente y social y la universidad no ha salido ilesa de estos cambios, ya no es la "torre de marfil" y pasó a involucrarse y devolver a la sociedad en su entorno (Giménez, 2017).

Está en curso una revolución en las universidades, en la cual el desarrollo socioeconómico pasa a integrar una de las misiones de la emergente universidad empresarial, fruto del aumento de la relevancia del conocimiento y de la investigación para el desarrollo (Etzkowitz, 1998, Etzkowitz y Leydesdorff, 2000, Rolfo y Finardi, 2014, Sam y Van der Sijde, 2014). La universidad es una referencia cultural de la sociedad y pasa a ser vista, también, como una prestadora de servicios (Hespanhol Bernardo, 2014).

En las últimas décadas, las universidades fueron presionadas para contribuir a demandas de la sociedad con un papel más activo (Giménez, 2017, Holmén y Ljungberg, 2015, Romero et al., 2016). Dejaron de ser instituciones que analizan y contemplan los problemas sociales y partieron para un compromiso que genere soluciones alternativas para transformar las condiciones que impiden el desarrollo local, provincial o nacional (Hernández Aramburo, González Cruz y Ceja Rivas, 2017).

Son universidades que van más allá de sus tareas tradicionales, avanzando en transferencia de tecnologías e innovaciones (Secundo, Perez, Martinaitis y Leitner, 2017), capaces de asumir la responsabilidad frente la sociedad y al sistema de innovación (Sam y Van der Sijde, 2014). Ellas 
deben involucrarse en las actividades de emprendimiento e innovación asumiendo un papel central en el sistema de producción y la transferencia de conocimiento (Minola, Donina y Meoli, 2016, Sam y Van Der Sijde, 2014).

Las prácticas comerciales de la universidad se centran en la negociación de patentes, la concesión de licencias, la transferencia de tecnología, en las incubadoras y en los parques científicos (Abreu, Demirel, Grinevich y Karataş-Özkan, 2016, Guerrero y Urbano, 2014). Sin embargo, los efectos de esta transferencia de tecnología y compartir el conocimiento por parte de la universidad, representando aportes directos al medio empresarial, todavía rinde debates entre aquellos que defienden la enseñanza y la investigación tradicionales (Etzkowitz y Leydesdorff, 2000).

Aceptar como excluyentes las relaciones entre patentes o publicaciones, investigación básica o aplicada colocan el avance científico fuera de los carriles de la tecnología y este escenario ya no es coherente (Etzkowitz, 1998, Brasil, 2015). La distancia entre las universidades públicas brasileñas y el medio empresarial es uno de los motivos para no figuraren en la vanguardia a nivel mundial (Brasil, 2015). Wang et al. (2016) muestran que esta interacción proporciona flexibilidad, autonomía y también beneficios a la enseñanza y la investigación, siendo positiva tanto para la Universidad y para la comunidad de negocios.

Una prueba del impacto positivo de la universidad en el desarrollo local es la aparición de negocios innovadores en sus proximidades geográficas, una vez que ellas favorecen el intercambio de conocimientos y tecnología (Calcagnini, Favaretto, Giombini, Perugini y Rombaldoni, 2016). La simbiosis entre las universidades y las empresas se define por Calcagnini et al. (2016) como generadora de innovación y actividad de emprendimiento y requiere proximidad y contactos directos entre los empresarios, los investigadores y los graduados.

\section{Las Misiones de la Universidad}

En términos generales, entre las misiones de la universidad, a la enseñanza cabe formar profesionales mediante transmisión del conocimiento científico, la investigación se centra en la producción de nuevos conocimientos expandiendo el saber humano y la vinculación o extensión compite articular el conocimiento de la universidad en la sociedad, con el propósito de socialización (Santos, 2014).

La enseñanza es el principal papel de la universidad, educando a la población y preparando personal calificado para el mercado de trabajo (Molas-Gallart et al., 2002, Rays, 2012, Wang et al., 2016). Es la base teórica necesaria para soportar las actividades de extensión (Infante y Miranda, 2015), una vez que, segundo Freire (1996), la enseñanza no es transferir conocimiento, sino crear condiciones para la construcción de 
conocimiento, sea por medio de diálogos, de problematizaciones, experiencias prácticas, inquietudes e indagaciones. Enseñar significa administrar las variables del contexto, de los contenidos, de las peculiaridades de los grupos de alumnos, variables que juntas componen el proceso de aprendizaje (Zabalza, 2007).

En la universidad moderna empezó la misión de investigación, junto con la enseñanza, conocida como la primera revolución en la universidad en 1810 (Etzkowitz, 1998, Etzkowitz y Leydesdorff, 2000, Gomes, Nogueira Neto, Barros y Sousa, 2017, Rolfo y Finardi, 2014, Sanchez-Barrioluengo, 2014, Wang et al., 2016). La investigación académica es instigada por la curiosidad, es conocida por promover el conocimiento y resolver los problemas (Molas-Gallart et al., 2002). Según Calcagnini et al. (2016), la investigación académica puede difundir el conocimiento académico por medio de la publicación de sus resultados en periódicos y de esta forma socializar a bajo costo y a los más lejanos interesados en su contenido.

La vinculación universitaria inició en Inglaterra en 1850, llegando después a América, caracterizada por la formación de recursos humanos y entrenamientos, cuando docentes de Oxford y Cambridge se desplazaban hasta las comunidades en el entorno para extender el conocimiento universitario por medio de charlas sobre literatura, cuestiones sociales y agrícolas (Gimenez, 2017). La extensión universitaria en Brasil tuvo su inicio en 1912, según el Foro de Pro-Rectores de Extensión de las Universidades Públicas Brasileñas [FORPROEX] (2012), con cursos de extensión y prestación de servicios en la Universidad Libre de São Paulo y en la Escuela Superior de Agricultura Veterinaria de Viçosa. Eran acciones con carácter asistencialista, descomprometidas con la transformación de la realidad (Anjos, 2014).

La extensión precisa ampliar la visión tradicionalista hacia relaciones más amplias, complejas y activas de la universidad en la sociedad, aunque persistan algunos signos de asistencialismo (Gimenez, 2017). En Brasil, no todas las universidades reconocen las actividades ligadas a la innovación, a la transferencia de tecnología y a la propiedad intelectual como vinculación, tal vez por desconocimiento, prejuicio con la relación más cercana con la sociedad empresarial, preferencias por acciones dirigidas a la población más carente o por acciones culturales (Gimenez, 2017).

Calcagnini et al. (2016) enumeran algunas de las actividades de la tercera misión: patentes, acuerdos de colaboración, spin-offs y las incubadoras de empresas. Estas y más consultorías, investigaciones contratadas y comercialización de la investigación académica son listadas por Holmén y Ljungberg (2015) como interacciones con la industria dentro de la tercera misión, idea compartida con Wang et al. (2016). 
La relación entre universidad y sociedad va mucho más allá de meras actividades comerciales, llegando a contribuciones a los gobiernos, los servicios públicos y la sociedad civil (Molas-Gallart et al., 2002, Simões, 2016). Además de los efectos positivos para la sociedad, la interacción promovida por la extensión es importante en la formación del académico, sea, por ejemplo, comercializando sus investigaciones y patentes o abriendo una empresa (Holmén y Ljungberg, 2015, Rosli y Rossi, 2016). Según Hernández Arámburo, Cruz González y Ceja Rivas (2017), combinar la extensión a la enseñanza y a la investigación potencializa el aprendizaje por promover situaciones, ambientes y metodologías diversificadas y contextualizadas. Se aproxima el estudiante de la práctica profesional mucho antes de la conclusión de sus estudios (Estrada, Rejas y Urías, 2016).

Las actividades de vinculación todavía no reciben la misma valoración que la enseñanza y la investigación, según las críticas de Ricci y Civitillo (2017), refiriéndose a la realidad de las universidades italianas, que raramente declaran las acciones de vinculación en sus informes sociales, ya que la cualificación de la universidad se mide principalmente por su actuación en la enseñanza y la investigación.

De la misma forma, Santos (2014) afirma que la extensión es reconocidamente el eslabón más débil del trípode que forma con la enseñanza y con la investigación, tal vez por ser la función más "joven", o por ser considerada suplementaria o por la falta de recursos. La CAPES, fundación del Ministerio de Educación (MEC) en Brasil que hace acciones para el desarrollo de la investigación y es una de las principales fuentes de apoyo de la educación superior en Brasil, no exige que el docente actúe en la extensión (Coordenação de Aperfeiçoamento de Pessoal de Nível Superior [CAPES], 2008).

\section{Selección de Docentes Universitarios}

La calidad de la universidad refleja las características de su cuerpo docente y esto justifica la preocupación con la selección y la evaluación del desempeño de los maestros que garanticen servicios de alto nivel. La selección y la evaluación de desempeño buscan evaluar la capacidad de los docentes en producir los efectos que la universidad pretende, es decir, enseñanza, investigación y vinculación (Casillas Martín, Cabezas Gonzáles y Pinto Llorente, 2015).

La selección del profesorado universitario está entre las principales responsabilidades del sector de gestión de personal, ya que este proceso afecta el rendimiento de la universidad (Lotta, 2002, Rana y Malik, 2017). Posición un tanto diferente de Chiavenato (2014), que, a su vez, distribuye las responsabilidades entre el sector de la gestión de personas y el departamento que ordena el nuevo profesor, ya que considera que es muy 
importante participar en todo el proceso, para aumentar las posibilidades de éxito e integración del seleccionado.

La selección de personas debe estar de acuerdo con los objetivos de la institución (Rodrigues y Veloso, 2013). Hay discrecionalidad a los miembros de los comités responsables por el proceso de selección de los maestros con respecto a los criterios y méritos para ser elegido para la evaluación, y hoy en día no existe una definición explícita de lo que sería el mejor criterio para la selección de candidatos al cuerpo docente (Buela, 2007).

El acceso a la carrera de docente en universidades públicas brasileñas se realiza por medio de concurso público y el proceso de selección que predomina es la evaluación del currículo, las pruebas de conocimiento específico y la prueba didáctica (Cunha, Zanchet y Ribeiro, 2013). Las principales características evaluadas en los procesos de selección de docentes son: inteligencia, aptitud, conocimiento de la materia, didáctica, capacidad para cumplir las funciones inherentes a un docente universitario, experiencias en el magisterio y en investigaciones (Casillas Martín, Cabezas Gonzáles y Pinto Llorente, 2015). Este sistema es criticado pues los resultados de los concursos pueden variar en función de la banca de evaluación (Buela, 2007) y entiende que es posible operar un sistema de selección del profesorado universitario con preguntas definidas que reflejen las necesidades de la universidad de forma que sea más representativa en la producción científica mundial y para formar profesionales de excelencia.

Los funcionarios de una institución son activos valiosos y sus conocimientos, habilidades y actitudes representan las herramientas a través de las cuales las universidades pueden cumplir sus objetivos institucionales (Teimouri, Jenab, Moazeni y Bakhtiari, 2017). Son los docentes que están al frente de los conocimientos científicos, sociales, culturales y profesionales socializados con la sociedad (Simões, 2013). Un docente competente ayuda a la institución para alcanzar más fácilmente sus objetivos (Gurgel et al., 2018) siendo así una ventaja competitiva (Chiavenato, 2014).

Para seleccionar bien al candidato a docente, las características del cargo y del candidato tienen que ser bien definidas, objetivas y claras (Chiavenato, 2014). Por ejemplo, se muestra en la investigación hecha por Vargiu (2014) como los sistemas de selección de las universidades no fomentan los profesores a involucrarse con la comunidad, algo que va en la dirección opuesta a lo que se espera de los profesores en una universidad contemporánea. Hay una relación directa entre el proceso de selección de los docentes y la calidad de la educación superior que se anhela, pues lo que se exige en los concursos públicos representa lo que es importante para el desempeño de los futuros docentes (Cunha, Zanchet y Ribeiro, 2013). 


\section{Metodología}

El presente proyecto tiene un enfoque cualitativo con la explotación del material generado de la revisión bibliográfica. En la etapa inicial se realizó la investigación bibliométrica, seguida del análisis sistémico que culminaron en el corpus final, compuesto por los artículos más recientes sobre el tema y los autores de base. En esta exploración fue posible extraer los elementos principales del contenido para desarrollar su análisis. La comprensión de las bibliografías se realizó basándose en el análisis de contenido de Bardin (2008), siempre considerando si las fuentes de investigación contenían realmente las informaciones buscadas para este estudio.

La etapa de la categorización utilizó criterios cuantitativos y cualitativos para la generación de las categorías que sirvieron de base para analizar los instrumentos (convocatorias de docentes universitarios). Para la definición de las categorías de contexto se consideraron los objetivos de la investigación y principalmente el contenido analizado en la revisión de literatura. La expresión elegida para la categoría de contexto fue "la vinculación en los procesos de selección del docente universitario". La expresión seleccionada para esta única categoría atendió a todos estos requisitos y fue extraída del análisis cualitativo realizada por medio de la lectura del referencial bibliográfico de este trabajo.

Extraídos de la combinación de análisis cuantitativos y cualitativos, las categorías de análisis, las unidades de registro y de contexto representan el desmembramiento de la categoría de contexto. El análisis cuantitativo se basa en la cuenta de la frecuencia de palabras en el contenido de todo el referencial bibliográfico a través del software AtlasTi ${ }^{\circledR}$. La frecuencia de los términos, combinada con un análisis cualitativo realizado durante la lectura de los textos (inferencia), dio soporte a la definición de las categorías de análisis y de las unidades de registro y de contexto. La Tabla 1 presenta todas las categorías y unidades del análisis de contenido realizado.

Por lo tanto, las categorías de análisis quedaron así definidas: "transferencia de conocimiento para la sociedad" y "desarrollo tecnoeconómico regional". Esas son categorías de actividades de vinculación presentes en la universidad contemporánea, según la literatura actual y por eso deben ser contempladas en los procesos de selección de los docentes.

El mismo procedimiento se siguió para llegar a las unidades de registro. Después de la lectura de la cartera de referencias se esbozaron unidades de registro y el análisis cuantitativo canalizó estas elecciones. Las unidades de registro representan las perspectivas para las universidades contemporáneas, relacionadas con la vinculación, según lo que fue levantado en la revisión bibliográfica: 1) proyectos culturales, 2) proyectos buscando alternativas para problemas sociales, 3) cuestiones del medio ambiente, 4) espíritu empresarial, 5) spin- off, 6) incubadora, 7) licencias y 8) innovación. 
La categoría de contexto, las categorías de análisis y las unidades de registro son las ideas de este trabajo de manera categorizada y sirvieron de base para el análisis de las convocatorias de los docentes de la Universidad Tecnológica Federal del Paraná y de la Universidad del estado de Campinas, la mejor universidad de América Latina según el ranking de la Times Higher Education (https://www.timeshighereducation.com/, recuperado el 24, julio, 2018).

El análisis documental desarrollado se centra en los documentos de las dos universidades públicas brasileñas, lo que prevé sus planes estratégicos y más precisamente la última convocatoria de selección de docentes para la carrera de Ingeniería Mecánica encontrada en los sitios de recursos humanos de las universidades. 


\begin{tabular}{|c|c|c|c|}
\hline $\begin{array}{l}\text { Categoría de } \\
\text { contexto }\end{array}$ & $\begin{array}{l}\text { Categorías } \\
\text { de análisis }\end{array}$ & Unidades de registro & Unidades de contexto \\
\hline \multirow[t]{8}{*}{$\begin{array}{l}\text { La vinculación } \\
\text { en los procesos } \\
\text { de selección del } \\
\text { docente } \\
\text { universitario }\end{array}$} & \multirow[t]{3}{*}{$\begin{array}{l}\text { Transferencia } \\
\text { de } \\
\text { conocimiento } \\
\text { para la } \\
\text { sociedad }\end{array}$} & Proyectos culturales & $\begin{array}{l}\text { Los proyectos de arte y cultura (danza, teatro, técnicas circenses, performance) que contribuyen a } \\
\text { transformar el ambiente universitario en polo de producción y circulación artística erudita y popular, } \\
\text { formación, capacitación y cualificación de personas que actúan en el área y en interlocución con la } \\
\text { comunidad, puntos de cultura, movimientos sociales, la sociedad civil organizada y la producción cultural } \\
\text { local (MEC, 2015, FORPROEX, 2007) }\end{array}$ \\
\hline & & $\begin{array}{l}\text { Proyectos } \begin{array}{r}\text { buscando } \\
\text { alternativas } \\
\text { problemas sociales }\end{array} \\
\end{array}$ & $\begin{array}{l}\text { Interrelacionar la Universidad con los demás sectores de la sociedad, con vistas a una actuación } \\
\text { transformadora, orientada a los intereses y necesidades de la mayoría de la población y propiciar el } \\
\text { desarrollo social y regional, así como el perfeccionamiento de las políticas públicas (FORPROEX, 2012) }\end{array}$ \\
\hline & & $\begin{array}{l}\text { Cuestiones del medio } \\
\text { ambiente }\end{array}$ & $\begin{array}{l}\text { Implementación y evaluación de procesos de educación ambiental de reducción de la contaminación del } \\
\text { aire, aguas y suelo; discusión de la Agenda } 21 \text {; discusión de impactos ambientales de emprendimientos y } \\
\text { de planes básicos ambientales; preservación de recursos naturales y planificación ambiental; el medio } \\
\text { ambiente y la calidad de vida (FORPROEX, 2012) }\end{array}$ \\
\hline & \multirow[t]{5}{*}{$\begin{array}{l}\text { Desarrollo } \\
\text { tecno- } \\
\text { económico } \\
\text { regional }\end{array}$} & Espíritu empresarial & $\begin{array}{l}\text { Programas y proyectos, asesoría, consultoría y realización de eventos relativos a la constitución y gestión } \\
\text { de empresas juveniles, pre-incubadoras, incubadoras de empresas, parques y polos tecnológicos, } \\
\text { cooperativas y emprendimientos solidarios y otras acciones dirigidas a la identificación, aprovechamiento } \\
\text { de nuevas oportunidades y recursos de manera innovadora, con foco en la creación de empleos y negocios } \\
\text { estimulando la proactividad, formación, capacitación y calificación de personas que actúan en el área } \\
\text { (FORPROEX, 2007) }\end{array}$ \\
\hline & & Spin- off & $\begin{array}{l}\text { Es una forma de transferir tecnología de la universidad a la sociedad. Se produce cuando un conocimiento } \\
\text { generado en la universidad es incorporado en productos o servicios de una nueva empresa y ofrecidos al } \\
\text { mercado (Etzkowitz y Leydesdorff, 2000) }\end{array}$ \\
\hline & & Incubadora & $\begin{array}{l}\text { Espacio creado para facilitar que las tecnologías generadas en la universidad se transformen en negocios, } \\
\text { con soporte de entrenamientos, de acompañamiento y apoyo en la inserción en redes de conexión en el } \\
\text { ecosistema de innovación (Asociación Nacional De Entidades Promotores De Empleo Innovadores, 2016) }\end{array}$ \\
\hline & & Licencias & $\begin{array}{l}\text { Es la autorización que el propietario de la patente concede a alguna empresa para fabricar y comercializar } \\
\text { el producto patentado, normalmente mediante un contrato formalizado entre las partes (Organización Para } \\
\text { Cooperación Y Desarrollo Económico [OCDE], 2005) }\end{array}$ \\
\hline & & Innovación & $\begin{array}{l}\text { Es la implementación de un producto (bien o servicio) nuevo o significativamente mejorado, o un proceso, } \\
\text { o un nuevo método de marketing, o un nuevo método organizativo en las prácticas de negocios, en la } \\
\text { organización del lugar de trabajo o en las relaciones externas (OCDE, 2005). }\end{array}$ \\
\hline
\end{tabular}

Tabla 1 - Definición de las categorías de contexto, categorías de análisis, unidades de registro y unidades de contexto 


\section{Resultados}

\section{Universidad Tecnológica Federal del Paraná (UTFPR)}

Se procedió al análisis de la última convocatoria de docentes para la carrera de Ingeniería Mecánica de la UTFPR (Edicto 010/2017-CPCP-CTProfesor de la Enseñanza Superior) en busca de puntos que exigieran del candidato competencia en el intercambio del conocimiento junto a la sociedad y en el desarrollo tecnológico y económico de la región.

En cuanto a la estructura, el edicto de convocatoria evidencia prueba escrita, desempeño docente, producción intelectual y títulos. La ponderación atribuida a cada una de ellas fue, respectivamente, 2, 3, 2 y 3 (Universidade Tecnológica Federal do Paraná [UTFPR], 2017a).

La prueba escrita fue eliminatoria y buscó evaluar la capacidad del candidato de disertar sobre uno de los temas que componen el contenido del programa. Se tomó en consideración la capacidad analítica y crítica del tema, la complejidad del texto elaborado, la articulación de los contenidos, la claridad de las ideas y de los conceptos y la corrección ortográfica. La nota mínima en esta prueba fue 50 puntos (UTFPR, 2017a).

La prueba de desempeño docente fue aplicada para los clasificados en la prueba escrita. Se evaluó el conocimiento, la habilidad y la actitud del candidato durante una clase de hasta treinta minutos para la banca de evaluadores. El tema de la clase fue sorteado un día antes. En el análisis de las pruebas escritas y de desempeño de enseñanza se observa que están dirigidas exclusivamente a evaluar el desempeño del candidato en la enseñanza, y juntas representan el 50\% de los puntos que el candidato puede alcanzar (UTFPR, 2017a).

En la prueba de producción intelectual, el candidato expuso su producción intelectual y tema de investigación y la banca argumentó en cuanto a la adherencia del tema de investigación al área del concurso, dominio del tema, relación de éste con las investigaciones del campus, interacción con otros grupos de investigación y claridad. La prueba de producción intelectual evalúa la adherencia del área de investigación del candidato y tampoco reconoce ninguna actuación en la vinculación (UTFPR, 2017a).

Para la prueba de títulos se convocaron todos los candidatos aprobados en la prueba escrita. Esta etapa del proceso selectivo reconoció, en un primer grupo, publicaciones de artículos, de libros, de capítulos de libros, de trabajos y patentes registradas y concedidas. Este grupo representa 80 de los 100 puntos posibles. En el segundo grupo, con límite en 20 puntos, se consideran proyectos financiados por organismos públicos brasileños, como CAPES y Consejo Nacional de Desarrollo Científico y Tecnológico (CNPq), orientación de maestría y doctorado, participación en bancas, experiencia en el magisterio superior y en otras profesiones en el área del concurso 
(UTFPR, 2017a). En esta prueba de títulos se observa que más del 80\% de los puntos reflejan actividades relacionadas a la investigación y que el resto de los puntos corresponde a actividades de enseñanza y experiencia profesional, en el magisterio y fuera de él.

Entre las categorías planteadas en este estudio y que sirvieron de base para este análisis, se identificó que no se considera la experiencia del candidato a docente de la UTFPR en la vinculación, sea en el compartir del conocimiento por medio de proyectos culturales, de alternativas a los problemas sociales y el medio ambiente, o experiencia empresarial, spin- off, incubadora, concesión de licencias y la innovación.

Este resultado revela un desajuste entre lo que la UTFPR especifica en su Plan de Desarrollo Institucional 2018-2022 y lo que hace, ya que establece que la vinculación ocurra de forma inseparable de la educación y la investigación para fortalecer los vínculos entre el conocimiento académico y los problemas reales de la comunidad y, por ser una universidad tecnológica, que debe mantener una fuerte interacción con el sector productivo (UTFPR, 2017b).

La selección de personas debe estar de acuerdo con los objetivos de la organización, todavía queda aparente que no hay relación entre las competencias exigidas del candidato y los objetivos de la UTFPR como una universidad contemporánea que busca promover una formación transitando entre enseñanza, investigación y tercera misión. Se constata con este análisis que la tercera misión no es relevante en el proceso de selección de docentes de la UTFPR, ya que en ninguna de las cuatro etapas del proceso la vinculación es considerada. El candidato tiene dos etapas dirigidas exclusivamente a la enseñanza y otras dos predominantemente relacionadas con la investigación.

\section{Universidad del Estado de Campinas (Unicamp)}

El análisis del edicto de convocatoria de docentes de Ingeniería Mecánica de la Unicamp (Proceso 03-P-29317/2016) permitió explorar su contenido para fines de comparación con las unidades de registro extraídas del análisis de contenido. La última convocatoria de la Unicamp para la selección de profesores estableció un perfil deseable para el candidato en el área del concurso, con características como:

- licenciatura y / o posgrado,

- capacidad para impartir cursos de graduación,

- potencial para desarrollar proyectos de investigación,

- competencia para orientar postgraduados y alumnos de iniciación científica, y

- actuación, preferentemente, en las áreas del concurso. 
Este proceso se estructuró con la prueba escrita, títulos, prueba didáctico $\mathrm{y}$ argumentación, con peso de 1 cada una (Universidade Estadual de Campinas [Unicamp], 2016a). En la prueba escrita el candidato necesitó discurrir sobre un asunto relativo al contenido del programa de disciplinas del concurso. Fue la única etapa que podía ser eliminatoria, pues si tenían más de tres candidatos, pasarían al siguiente paso aquellos que recibieran la puntuación mínima de 7,0 en tres de los cinco jueces.

La prueba de títulos vino en la secuencia para evaluar el memorial presentado por el solicitante que contiene las actividades realizadas, los trabajos publicados y otras informaciones que le confieren mérito como títulos universitarios, currículum vitae et studiorum, actividades científicas, didácticas y profesionales, títulos honoríficos, becas de estudio a nivel de postgrado y participaciones en cursos, congresos, simposios y seminarios (Unicamp, 2016a). Esta podría ser una oportunidad de verificar si, entre las experiencias presentadas en su currículo, constan algunas relacionadas con la vinculación.

La penúltima etapa fue la prueba de argumentación, donde la comisión juzgadora del proceso selectivo cuestionó el solicitante sobre los temas del programa del curso y sobre las actividades citadas en el memorial (Unicamp, 2016a). Esta sería otra oportunidad de identificar en el candidato su interés por la tercera misión. La prueba de títulos y la argumentación, juntas, suman el 50\% de la nota del candidato. En el proceso selectivo de docente Ingeniero Mecánico, según la lista de requisitos deseados del candidato, la actuación en la vinculación no consta como una de las capacidades esperadas. De esta forma, se lleva a creer que en estas dos etapas los evaluadores se centraron principalmente en las capacidades relacionadas con la enseñanza y la orientación de graduación y posgrado y con los proyectos de investigación en las áreas del concurso.

La prueba didáctica cerró las etapas de selección con una exposición oral del candidato, durante cincuenta o sesenta minutos, para los miembros de la banca, sobre materia sorteada el día anterior (Unicamp, 2016a). En un análisis de las pruebas escrita y didáctica del edicto para docente Ingeniero Mecánico, se nota que están dirigidas exclusivamente para evaluar la competencia del candidato en la enseñanza, y juntas representan el 50\% de los puntos que el candidato puede alcanzar (Unicamp, 2016a).

El resumen del análisis realizado en este edicto de selección de docente para el curso de Ingeniería Mecánica de la Unicamp, es que no se encontraron, de forma explícita, las categorías levantas en este trabajo, relativas a la extensión. La experiencia de candidato en proyectos culturales, proyectos para los problemas sociales, proyectos en materia ambiental, el espíritu empresarial, spin- off, incubadora, concesión de licencias y la innovación pueden ser que sean puntuadas en las pruebas de títulos y en la 
argumentación, pero no hay como estar seguros. Además, si la actuación en la tercera misión no consta en la lista de capacidades deseadas en el candidato, se espera que la banca se base en la puntuación atribuida en actividades relacionadas solamente con la enseñanza y la investigación.

Este resultado va de encuentro con lo que establece la Planificación Estratégica 2016-2020 de la universidad, por debilitar a educación idealizada prevista para que sea inseparable entre la enseñanza, investigación y vinculación. La Unicamp pretende expandir, en este período, las acciones de tercera misión en busca de soluciones para los problemas regionales (Unicamp, 2016b). Rodrigues y Veloso (2013) advierten que la selección de personas debe estar de acuerdo con los objetivos de la institución.

Se concluye con este análisis que la vinculación no es relevante en la selección de los profesores de la Unicamp, ya que no se requiere del candidato un mínimo de experiencia en la extensión. Las etapas del proceso requieren que el candidato combine competencias relacionadas con la enseñanza y la investigación.

\section{Discusión}

Los resultados apuntan a una menor relevancia de la vinculación en los procesos de selección, en las dos universidades brasileñas seleccionadas para este estudio, sin embargo, la complejidad del papel de la universidad en la sociedad va más allá de la enseñanza y la investigación tradicional (Etzkowitz, 1998, Etzkowitz y Leydesdorff, 2000, Sam y Van der Sijde, 2014).

Como afirmó Gimenez (2017), a lo largo del tiempo la sociedad como un todo evolucionó y la universidad no pasó ilesa por esto, dejó de ser la "Torre de Marfil" y pasó a involucrarse con la sociedad. Esta participación con la sociedad es la tercera misión de la universidad y las recientes transformaciones exigen un papel destacado de estas instituciones en la sociedad (Gimenez, 2017, Vargiu, 2014), lo que el resultado de este estudio contradice.

Se está en curso una revolución en las universidades, en la cual el desarrollo socioeconómico pasa a integrar uno de sus focos (Etzkowitz, 1998, Etzkowitz y Leydesdorff, 2000, Rolfo y Finardi, 2014, Sam y Van Der Sijde, 2014), sin embargo, entre las acciones de vinculación relacionadas con el desarrollo tecno-económico regional, planteadas en este trabajo, sólo los licenciamientos de patentes aparecieron en los procesos de selección de la Unicamp y ninguna en la UTFPR. De esta forma resulta difícil atender a las demandas socioeconómicas de la sociedad actual, apuntada como uno de los objetivos de la universidad (Minola, Donina y Meoli, 2016, Rolfo y Finardi, 2014, Rosli y Rossi, 2016, Sam y Van der Sijde, 2014, Santos, 2005, Santos, 2014, Wang et al., 2016). 
Esta aparente separación de las actividades relacionadas con el desarrollo regional, constatada en las dos universidades brasileñas, por no asignar relevancia adecuada a la vinculación universitaria en los procesos de selección de docentes, va en contra de lo que previó el Plan Nacional de Educación brasileño 2001-2010, que indicó un acercamiento entre las universidades y las empresas en la mejora de vida de la sociedad y en la competitividad del país (Gomes et al., 2017). Como señaló Gimenez (2017), esto sería una diversificación de la actuación de la universidad al promover la innovación y el desarrollo tecnológico. Por la teoría de la Triple Hélice la universidad puede ser una fuerza motriz de la innovación (Etzkowitz y Leydesdorff, 2000).

Al no valorar las acciones de los maestros en el mundo empresarial, las spin- offs, las incubadoras, la concesión de licencias y la innovación, la Unicamp y la UTFPR revelan una cierta falta de alineación con otras partes interesadas en la región para facilitar el desarrollo, como se ha señalado (Secundo et al., 2017). También contraria Minola, Donina y Meoli (2016), Sam y Van der Sijde (2014), que defienden la universidad con un papel central en el sistema de producción de conocimiento, que participan en actividades de emprendimiento e innovación.

\section{Conclusión}

En el análisis de los edictos de convocatoria de docentes de la Unicamp y de la UTFPR, fue posible alcanzar el objetivo general de este trabajo que era demostrar la relevancia de las misiones de la universidad, en la perspectiva de la universidad contemporánea, en los procesos de selección de los docentes.

En los procesos de selección de docentes de Ingeniería Mecánica de la Unicamp y la UTFPR analizados en este estudio, estaba claro que hay menor relevancia de la vinculación en relación a la enseñanza y la investigación. El candidato a docente necesita demostrar experiencia sólo en la enseñanza y la investigación. Seleccionado sin sesgo extensionista, el docente de Ingeniería Mecánica tiende a seguir una carrera apoyada solamente en la enseñanza y la investigación. Es éste el perfil de docente que estas instituciones seleccionan, incluso la vinculación siendo una de las tres misiones esenciales de la universidad. Posiblemente este problema no sea exclusivo de estas dos universidades en Brasil.

El énfasis de la universidad contemporánea está en su tercera misión. Combinada con la enseñanza, ella colabora con la didáctica de las actividades prácticas, o con la aplicación de los conocimientos de la investigación e inserta cuestiones reales de la sociedad en la formación del estudiante. Sus proyectos pueden resolver problemas sociales con el 
compartir el conocimiento académico, así como pueden reflejar en el desarrollo tecnológico y económico.

Son muchas las contribuciones de la vinculación universitaria a la sociedad. Sin embargo, las universidades analizadas en este trabajo, con respecto a la carrera de Ingeniería Mecánica, no demuestran la debida valorización de la actuación docente en este frente, equivalente a la importancia dada a la enseñanza y a la investigación.

\section{References:}

1. Abreu, M., Demirel, P., Grinevich, V. y Karataş-Özkan, M. (2016). Entrepreneurial practices in research-intensive and teaching-led universities. Small Business Economics, 47, 695-717.

2. Anjos, M.C.R. (2014). Fronteiras na construção e socialização do conhecimento científico e tecnológico: um olhar para a extensão universitária (tesis doctoral). Universidade Federal de Santa Catarina, Florianópolis, SC, Brasil.

3. Asociación Nacional De Entidades Promotores De Empleo Innovadores. (2016). Estudo de impacto econômico: segmento de incubadoras de empresas do Brasil. Brasília, DF: Autor, SEBRAE. Recuperado el 6, junio, 2018 de http://www.anprotec.org.br/Relata/18072016\%20Estudo_ANPROTE C_v6.pdf

4. Bardin, L. (2008). Análise de Conteúdo. Lisboa: Edições 70.

5. Coordenação de Aperfeiçoamento de Pessoal de Nível Superior [Capes] (2008, junio). História e Missão. Recuperado de http://www.capes.gov.br/historia-e-missao

6. Buela, G. (2007). Reflexiones sobre el sistema de acreditación del profesorado funcionario de Universidad en España. Psicothema, 19(3), 473-482.

7. Calcagnini, G., Favaretto, I., Giombini, G., Perugini, F. y Rombaldoni, R. (2016). The role of universities in the location of innovative start-ups. Journal of Technology Transfer, 41, 670-693.

8. Cascio, W. F. (2015) Managing human resources: productivity, quality of work life, profits (10 ed.) Nueva York: McGraw-Hill.

9. Casillas Martín, S., Cabezas González, M. y Pinto Llorente, A. M. (2015). Evaluación del profesor universitario: ¿A mayor categoría profesional mejor profesor? EKS, 16(3).

10. Chiavenato, I. (2014). Gestão de pessoas: o novo papel dos recursos humanos nas organizações (4 ed). São Paulo: Manole. 
11. Cunha, M. I., Zanchet, B. B. A. y Ribeiro, G. M. (2013). Qualidade do ensino de graduação: culturas, valores e seleção de docentes. Práxis Educativa, 8(1), 219-241.

12. D'Este, P., Tang, P., Mahdi, S., Neely, A. y Sánchez-Barrioluengo, M. (2013). The pursuit of academic excellence and business engagement: is it irreconcilable? Scientometrics, 95, 481-502.

13. Estrada, M. L. C., Rejas, M. J. R., Urias, E. U. (2016). Abrir las aulas: el vínculo entre docencia, investigación $\mathrm{y}$ vinculación comunitaria. Revista Brasileira de Educação, 21(66), 737-758.

14. Etzkowitz, H. (1998). The norms of entrepreneurial science: Cognitive-effects of the new university-industry linkages. Research Policy, 27, 823-833.

15. Etzkowitz, H. y Leydesdorff, L. (2000). The dynamics of innovation: from National Systems and 'mode 2' to a triple helix of universityindustry-government relations. Research Policy, 29(2), 109-123.

16. Fórum de Pró-Reitores de Extensão das Universidades Públicas Brasileiras [FORPROEX]. (2007). Extensão Universitária: organização e sistematização. Belo Horizonte: Coopmed.

17. Fórum de Pró-Reitores de Extensão das Universidades Públicas Brasileiras [FORPROEX]. (2012). Política nacional de extensão universitária. Manaus: Autor.

18. Freire, P. (1996). Pedagogia da autonomia: saberes necessários à prática educativa. São Paulo: Paz e Terra.

19. Gimenez, A. M. (2017). As multifaces da relação universidadesociedade e a construção do conceito de terceira missão (tesis doctoral). Universidade Estadual de Campinas, Campinas, SP, Brasil.

20. Gomes, W. V., Nogueira Neto, A., Barros, A. D. M. y Sousa, A. M., Jr. (2017). Entrepreneurial university: a study on the differences between Brazil and the model done in the United States. Espacios, $38(28), 32$.

21. Guerrero, M. y Urbano, D. (2014). Academics' start-up intentions and knowledge filters: An individual perspective of the knowledge spillover theory of entrepreneurship. Small Business Economics, 43(1), 57-74.

22. Gurgel, A. M., Pimenta, I. L., Santos, J. W. dos y Medeiros Júnior, J. V. de. (2018). Seleção de profissionais de TI por competências em uma instituição do ensino superior: uma proposta baseada em um modelo multicritério de apoio à decisão. Gestão \& Produção, 25(1), 16-29.

23. Hernandez Arámburo, R., Cruz González, H. y Ceja Rivas, A. (2017). University vinculación: A two-way strategy for sustainable 
development and academic relevance. Gateways: International Journal of Community Research and Engagement, 10, 14-32.

24. Hespanhol Bernardo, M. (2014). Produtivismo e precariedade subjetiva na universidade pública: o desgaste mental dos docentes. Psicologia \& Sociedade, 26, 129-139.

25. Holmén, M. y Ljungberg, D. (2015). The teaching and societal services nexus: academics' experiences in three disciplines. Teaching in higher education, 20(2), 208-220.

26. Infante, R. C. H. y Miranda, M. E. I. (2015). La formación humanística y humanista en los estudiantes universitarios. Revista digital de investigación en docencia universitaria, 9(2).

27. Lotta, G. S. (2002). Avaliação de desempenho na área pública: perspectivas e propostas frente a dois casos práticos. RAE-eletrônica, $1(2)$.

28. Ministério da Educação [MEC]. (2015 febrero). Edital PROEXT 2015 - Programa de Extensão Universitária MEC/SESu. Recuperado el 5, junio, 2018, de http://portal.mec.gov.br/index.php?option=com_docman\&view=dow nload\&alias=15149-edital-proext-2015\&category_slug=fevereiro2014\&Itemid $=30192$

29. Minola, T., Donina, D. y Meoli, M. (2016). Students climbing the entrepreneurial ladder: Does university internationalization pay off? Small Business Economics, 47, 565-587.

30. Molas-Gallart, J., Salter, A., Patel, P., Scott, A. y Duran, X. (2002). Measuring third stream activities. Final report to the Russell Group of University, Brighton: SPRU.

31. Organización Para Cooperación Y Desarrollo Económico [OCDE] (2005). Manual de Oslo. Diretrizes para coleta e interpretação de dados sobre inovação (3 ed).

32. Pires, C. y Rausch, R. B. (2016). Avaliação institucional na FEBE: contexto e perspectivas. Revista da UNIFEBE, 1(7), 7-14.

33. Rana, M.H. y Malik, M.S. (2017). Impact of human resource (HR) practices on organizational performance: Moderating role of Islamic principles. International Journal of Islamic and Middle Eastern Finance and Management, 10(2), 186-207.

34. Rays, O. A. (2012, Março). Ensino-Pesquisa-Extensão: notas para pensar a indissociabilidade. Revista Educação Especial, 71-85.

35. Ricci, P. y Civitillo, R. (2017). Accountability and third mission in Italian universities. International Journal of Managerial and Financial Accounting, 9(3). 
36. Rodrigues, A. F. y Veloso, A. Contribuições da Gestão de Recursos Humanos para a Criatividade e Inovação Organizacional. (2013). Revista Psicologia: Organizações e Trabalho, 13, 293-308.

37. Rolfo, S. y Finardi, U. (2014). University third mission in Italy: organization, faculty attitude and academic specialization. Journal of Technology Transfer, 39, 472-486.

38. Romero, L. E. A. et al. (2016). Vinculación comunitaria y diálogo de saberes en la educación superior intercultural en México. Revista Mexicana de Investigación Educativa, 21(70), 759-783.

39. Rosli, A. y Rossi, F. (2016). Third-mission policy goals and incentives from performance-based funding: are they aligned? Research evaluation, 25(4), 427-441.

40. Sam, C. y Van der Sijde, P. (2014). Understanding the concept of the entrepreneurial university from the perspective of higher education models. Higher Education, 68, 891-908.

41. Sánchez-Barrioluengo, M. (2014). Articulating the "three-missions" in Spanish universities. Research Policy, 43, 1760-1773.

42. Santos, B. S. (2005). A universidade no século XXI: para uma reforma democrática e emancipatória da universidade. (3 ed.). São Paulo: Cortez.

43. Santos, M. P. (2014). A extensão universitária como "laboratório" de ensino, pesquisa científica e aprendizagem profissional: um estudo de caso com estudante do curso de licenciatura em Pedagogia de uma faculdade particular do Estado do Paraná. Extensio: Revista Eletrônica de Extensão, 11(18), 36-52.

44. Secundo, G., Perez, S. E., Martinaitis, Z. y Leitner, K. H. (2017) An Intellectual Capital framework to measure universities' third mission activities. Technological Forecasting and Social Change, 123, 229239.

45. Shipton, H., West, M.A., Dawson, J., Birdi, K. y Patterson, M. (2006). HRM as a predictor of innovation. Human Resource Management Journal, 16(1), 3-27.

46. Simões, M. L. (2013). O surgimento das universidades no mundo e sua importância para o contexto da formação docente. Revista Temas em Educação, 22(2), 136-152.

47. Simões, M. A. S. C. (2016). A avaliação da extensão universitária nas Universidades Federais da Bahia (tesis doctoral). Universidade do Minho, Braga, Portugal.

48. Teimouri, H., Jenab, K., Moazeni, H.R. y Bakhtiari, B. (2017). Studying effectiveness of human resource management actions and organizational agility: Resource management actions and 
organizational agility. Information Resources Management Journal, $30(2)$.

49. Universidade Estadual de Campinas [Unicamp]. (2016a, diciembre). Processo 03-P-29317/2016. São Paulo: Diario Oficial, 165. Recuperado el 20 febrero, 2018, de https://www.sg.unicamp.br/dca/concursos/a/concursos-paraprofessor-doutor/faculdade-de-engenharia-mecanica

50. Universidade Estadual de Campinas [Unicamp]. (2016b). Planejamento Estratégico da Unicamp 2016-2020. Campinas, SP: Unicamp. Recuperado el 01 abril, 2018, de http://www.unicamp.br/unicamp/ju/650/planejamento-estrategico-daunicamp-2016-2020

51. Universidade Tecnológica Federal do Paraná [UTFPR] (2017a, Junho). Concursos Públicos para Docente Efetivo - Editais válidos. Edital 010/2017-CPCP-CT - Professor do Magistério Superior. $\begin{array}{lllll}\text { Recuperado el } & 18 & \text { Octubre, } & 2017 & \text { de }\end{array}$ http://www.utfpr.edu.br/concursos/campi/ct/cpcp

52. Universidade Tecnológica Federal do Paraná [UTFPR]. (2017b). Plano de Desenvolvimento Institucional da UTFPR - PDI 2018-2022. Curitiba: Autor. Recuperado el 01 abril, 2018, de http://portal.utfpr.edu.br/comissoes/consulta/consulta-publica-pdi2018-2022

53. Vargiu, A. (2014). Indicators for the evaluation of public engagement of higher education institutions. Journal of the Knowledge Economy, $5,562-584$.

54. Wang, Y., Hu, R., Li, W. y Pan, X. (2016). Does teaching benefit from university-industry collaboration? Investigating the role of academic commercialization and engagement. Scientometrics, 106, 1037-1055.

55. Zabalza, M. A. (2007). O ensino univesitário: seu cenário e seus protagonistas. Madrid: Artmed. 